\title{
Research on Embedded Software Design and Algorithm
}

\author{
Qing Wan \\ Chongqing Vocational Institute of Engineering, Chongqing, 402260
}

Keywords: Embedded, Software Design, Algorithm

\begin{abstract}
Embedded systems are the most common forms of application for computers in the post-PC era. This paper introduces the technical characteristics of a class of landmark embedded devices, analyzes the challenges and development trends of embedded software technology, and finally, puts forward some ideas for developing embedded software technology in China.
\end{abstract}

\section{Introduction}

In the rapid development of computer technology, a modern society, a single computer equipment has been difficult to meet people's life and production needs, people began to all types of embedded software put forward new requirements. In everyday life, embedded software can be seen everywhere. Network TV, smart appliances, automotive electronic control, GPS, smart phones, smart game consoles, etc. are all applications that use embedded software. People's daily life is inseparable from the embedded software technology and embedded software development direction will become the key to the future of production and life. Therefore, researching the status quo and development trend of embedded software has very important practical significance for the development and progress of embedded software technology.

Software is designed to meet the needs of the development of the procedures, documents and related technologies. From a classification point of view, the software can be divided into system software and application software, from the application range can be divided into database software, embedded software. From the computational theory point of view, the general software can be seen as a set of procedural mathematical functions, the function input data implicit output data. The embedded software is also a mathematical function, but its main role in embedded software is not to convert data, but with the physical field Unicom. Simply put, is to use the relevant technology to the operating system or other development software embedded in the hardware.

\section{Embedded software classification and development features}

Embedded operating system EOS (Embedded Operating System) is a very popular system software. In the past the operating system is more used in industrial production and defense management and control areas. Embedded operating system needs to complete all the hardware and software embedded in the system scheduling, adjustment, control and coordination. Embedded operating system must fully reflect the characteristics of the system, through the installation and uninstall module to complete the operation of the system. In general, embedded operating systems are more suitable for commercial use. Since the 1980s, commercial embedded operating systems have enjoyed a boom. Currently, the world's major embedded operating systems are Windows CE, Palm OS, Linux, Vx Works, etc. [1]. Relative to foreign countries, the development of China's embedded operating system started relatively late, and there is still some distance with foreign countries. Domestic products of this type are mainly implemented on Linux operating systems with independent copyright. Red Flag Linux and Oriental Linux are widely used. Embedded support software. The function of embedded support software is to assist the software development behavior and provide some help and support for software development. Embedded support software generally covers databases, commonly used development tools. Among them, the database is very crucial. At present, the application of embedded database technology has become very popular. At the same time, with the maturity of mobile Internet technology, people's demand for mobile data 
and requirements are also gradually increased. Embedded database technology has been in industrial production; the military sector has been given attention. The embedded mobile database is used to support the calculation of the database management system. The database system embedded in the mobile device involves different technical fields such as database technology, distributed computing technology and communication technology, and has become a field with strong research atmosphere and active progress in view of the current development status. Embedded application software is formed for a specific area, based on a specific hardware platform software, which can achieve the user and its goals. Due to the time and other details of user tasks, some embedded applications require a designated embedded operating system during the application process. Compared with the common software, the embedded application software has a very significant difference. It not only has higher requirements on accuracy, stability and security, but also optimizes the processing as much as possible to reduce the impact on the system Resource consumption, reduce hardware input costs. At present, there are various types of embedded applications on the market, commonly used embedded software browser, office processing software, multimedia software, human-machine control software and so on. Application software is the most active and active type in embedded systems. Each application has its own specified background. Although it is small in size, it has a high technical content. Therefore, the embedded application does not have the same operating system and support Software, like the monopoly of foreign products, but has a very broad development prospects. Therefore, embedded application software is the absolute advantage of embedded software in China.

Relative to the general computer software, embedded software development there is a certain particularity, mainly reflected in: First, the development of smaller, more difficult. The average size of the embedded software is relatively small, mostly within a few MB. Embedded software development needs to cover board-level initialization procedures, drivers, applications, and testing levels. Usually involves the development of low-level software [3]. Based on this demand, the development of embedded software is more difficult, and it needs to be done by various development technologies and development tools in the development process. In a few seconds after power on you need to enter the normal working environment. Therefore, most of the embedded software is often implemented in main memory such as Nor Flash, which can be started immediately after power-on. Or move directly into the memory Nor Flash run directly. Embedded software needs to react quickly to external events regardless of the current state of the system. At the same time, the ability to handle an asynchronous concurrent event and the ability to handle errors and automatically reset. Use fault tolerance, error handling means, when the software is running error or crash can be automatically restored to the original normal operation. Embedded software is a degree of integration is based on application and operating system integration program, so its development process needs to be integrated.

\section{Embedded software development and algorithms}

Embedded software is mainly formed on the host. Software developers use various types of tools to do editing, linking, etc., of applications to create programs that can be debugged or hardened. Relative to the general software development process, embedded software development is more special, its cross-compiler compiled on the host to form another embedded microprocessor binary program. At the same time, the cross linker will select the operating mode and memory location of the target program according to the technology and means of the host computer, adjust the corresponding information configuration, and the modules in the module object and the library file become the target program.

Embedded software debugging process requires the use of cross-debugger, the device that is required for debugging and debugging procedures, respectively, on different devices. Debugger according to the actual situation to use a certain way on the target machine to control the modulation object, and the target machine memory, registers and debugger object to verify and challenge. In the process of developing embedded software, cross-debugger is the most frequently used tools, often occupy software development time $30 \%-50 \%$. Therefore, the use of 
cross-debugger function and efficiency will have a direct impact on the embedded software technology development cycle and the market effect.

Embedded Software After debugging, the program code needs to be completely copied into the non-volatile memory in the paper target machine and run on the physical hardware environment. This process is the curing of embedded software. Therefore, the computer system will start the data migration to the computer's RAM, the program code will run in ROM, Flash Memory. Then the curing process will establish a Boot module. In the real physical target environment allows applications to work on the realization of the computer's central processor environment initialization. When the user opens the target machine, the application is automatically embedded into operation.

Compared with other common software development, embedded software does not exist the ability of self-development, in the process of embedded software development need to host and target each other to assign tasks. The host's main task is to compile, link, address and other processes, and the target machine is the actual operation of embedded software platform. First, the application needs to be transformed into a binary code that can work on the target machine. This whole process needs to be compiled, linked, and addressed. Compilation is achieved by cross-compiler. In the process of compiling the target file will be linked to the specified target file, the process is the link process. Addressing, on the other hand, assigns the physical memory geology to the target file at a relative offset. The document formed by this process is a binary file that can work in the embedded platform.

It can effectively improve the software threat, making software factory production play a positive role. Therefore, the application of IP component library technology in embedded software development has become more and more common. The best form of embedded system to achieve a single chip system (SOC, Systemon Chip), and the core of a single chip system is the IP core (Intelleetual Poperty Kernel S). Among them, IP core includes hardware core, software core and firmware core. Among them, the hardware core is the MPU core or DSP core, and the software core is a code unit that can modulate the standard API mode and the IP core component that are required by a single chip system into executable code units, and promote the embedded system of the SOC Development. At present, many embedded software manufacturers have already sold mature RTOS kernels and function extensions through IP core components. It can be seen that the developing and mature IP component software technology will provide space for the development of small and medium-sized high-tech software companies in the future.

\section{Conclusions}

The early 21st century, the world's mainstream computer technology has entered the post-PC era. After the era of PC, that is, non-PC information equipment supernatural era, represented by information appliances, is the main body of non-PC information equipment, its wide range of applications, prominent field characteristics, great room for development. This creates a good opportunity for the development of embedded software technology in our country. The worldwide expansion of Internet technology and the rapid development of China's telecommunications industry have created vast markets for the development of embedded products in our country. In addition, our country is a populous country and is well-known for software intellectual advantages. Therefore, our software colleagues, including the majority of western counterparts, should firmly grasp this great opportunity and identify the attack points. With the new round of embedded software technologies such as IP software, JAVA applications and wireless networks, some sprint, will be able to make our software technology to a higher level, to make due contributions to the development of China's economy.

\section{References}

[1] Huang Bai-Qiao. Hierarchical Construction of Embedded Software Fault Tree Based on IPO Functional Decomposition [J]. Journal of Shenyang Polytechnic University. 2013 (02) 
[2] Yin Yongfeng, Zheng Benxuan, Lu Minyan, Ni Hongying. Embedded software test case generation technology based on UML real-time extension [J]. Systems Engineering and Electronics. 2011 (03)

[3] Yang Fang, He Hongwei, Xie Peng, Rao Jinghong. Research and Design of Embedded Software Target Simulation Test Platform [J]. Computer Engineering and Design. 2009 (19)

[4] LUO Gang, GUO Bing, SHEN Yan, LIAO Haiyan, REN Lei. Analysis and Optimization of Power Consumption Characteristics of Source-level and Algorithm-level Embedded Software [J]. Journal of Computers

[5] Wang Yanna. Research and Implementation of Reusable Test Framework for Automatic Ticket Receiving Machine [J]. Shanghai Electric Power Institute. 2007 (04)

[6] Wang Heng. Research and implementation of a highly reliable remote self-updating mechanism of embedded software [J]. Industrial Control Computer. 2007 (09)

[7] Xiao Jian-yu, Zhang De-yun, Chen Hai-Quan, Dong Hao. High Confidence Embedded Software Development Based on UML State Machine and B Method [J]. Computer Engineering. 2006 (08) 